\title{
University students' sleep during an exam period: the role of basic psychological needs and stress
}

\author{
Rachel Campbell $^{1} \cdot$ Bart Soenens $^{1} \cdot$ Wim Beyers $^{1} \cdot$ Maarten Vansteenkiste ${ }^{1}$
}

๑) Springer Science+Business Media, LLC, part of Springer Nature 2018

\begin{abstract}
During exam periods university students are at risk for poor sleep. To understand variability in this vulnerability for poor sleep, the role of the satisfaction of the basic psychological needs for autonomy, competence, and relatedness was examined by assessing university students $\left(N=121 ; 78 \%\right.$ female; $M_{\text {age }}=21.69, S D=1.39$, range 19-25) before, during, and after an exam period. Need-based and sleep-related functioning deteriorated during the exam period and then improved after the exam period. As need satisfaction decreased (i.e., from pre-exam to exam period) sleep quality, sleep quantity and daytime functioning deteriorated, while subsequent increases in need satisfaction (i.e., from exam to the post-exam period) were accompanied by improvements in sleep quality and daytime functioning. These correlated changes in need-based experiences and the sleep-related outcomes were largely accounted for by changes in stress, suggesting that stress may play an explanatory role in these associations.
\end{abstract}

Keywords Self-determination theory $\cdot$ Basic psychological needs $\cdot$ Sleep $\cdot$ University students $\cdot$ Stress

\section{Introduction}

Emerging adulthood is conceptualized as a distinct developmental period during which youth often move away from home, begin higher education and parental supervision typically lessens (Arnett 2000), bringing with it new and unique challenges to sleep. Relative to adolescents, emerging adults who enter university generally have more freedom when setting bedtimes and more flexible class start times (Urner et al. 2009; Zimmerman 2011), both of which need to be managed in combination with increased academic demands. Furthermore, emerging adulthood is associated with increased risk for mental health problems (Blanco et al. 2008), which is also linked with sleep disturbance (Lund et al. 2010). As a result, sleep difficulties are highly prevalent among university students, with average estimates of sleep duration not reaching recommended levels (Doane et al. 2015) and 60\% of university students reporting poor sleep quality (Lund et al. 2010). Moreover, sleep has been shown to deteriorate

Rachel Campbell

Rachel.Campbell@UGent.be

1 Department of Developmental, Social, and Personality Psychology, Faculty of Psychology, Ghent University, Henri Dunantlaan 2, 9000 Ghent, Belgium during times when university students experience increased academic demands and stress, such as examination periods (Ahrberg et al. 2012; Zunhammer et al. 2014). Such sleep disturbances warrant attention, given that poor sleep is associated with poor academic functioning (Taylor et al. 2013) and has been put forward as a transdiagnostic risk factor for the development of psychopathology (Harvey 2008).

Previous research indicates that quality and quantity of sleep varies considerably among university students on a daily, monthly, and yearly basis (e.g., Galambos et al. 2009, 2010, 2013). However, only a few studies have examined factors that contribute to such within-person variation. The present short-term longitudinal study sought to examine the covariation between changes in the satisfaction of the basic psychological needs for autonomy, competence and relatedness, as conceived within Self-Determination Theory (SDT; Deci and Ryan 2000), and changes in university students' sleep-related functioning. In addition, by assessing participants' experienced stress before, during and after exposure to a common and potentially stressful event (i.e., an exam period) we also aimed to examine the intervening role of changes of stress in these dynamic associations. 


\section{Self-determination theory: basic psychological needs}

SDT is a macro-theory of human motivation that states that all human beings possess the inherent psychological needs for autonomy, competence, and relatedness, that when satisfied promote adaptive functioning (e.g. growth and well-being) and when frustrated yield negative effects (e.g., ill-being). Autonomy involves experiencing a sense of volition and self-endorsement in one's behavior. Competence involves feeling capable of achieving desired outcomes. Relatedness involves feeling close and connected with important others. When these needs are satisfied, people experience the freedom to be themselves (autonomy satisfaction), feel capable of dealing with daily tasks and challenges (competence satisfaction), and experience warmth and trust in their relationships (relatedness satisfaction) (Deci and Ryan 2000).

In accordance with the claim that these needs represent critical nutrients for wellness, their satisfaction has been shown to relate to higher vitality, self-esteem and life satisfaction (Deci and Ryan 2000). Such findings emerged among individuals from diverse cultural backgrounds and even among those who reported a low desire for or valuation of the satisfaction of these needs (Chen et al. 2015). Furthermore, these psychological needs are not only relevant to psychological well-being but are also important for physical outcomes such as sleep (Deci and Ryan 2000). For example, one cross-sectional study indicated that adults who experienced higher psychological need satisfaction over the past month, also reported better sleep quality, more adaptive daytime functioning as indexed by higher feelings of vitality and lower fatigue, and somewhat longer sleep duration (Campbell et al. 2015). Similar findings have been demonstrated among people living with HIV (Campbell et al. in press-a) and individuals with unexplained chronic fatigue (Campbell et al. 2017). Moreover, need-based experiences have also been shown to co-vary with subjective sleep quality and daytime functioning from day to day among both healthy (Campbell et al. 2018) and clinical samples (Campbell et al. in press-b). Most likely, these associations reflect reciprocal effects, with need satisfaction not only affecting sleep but with sleep also impacting on individuals' psychological need-based experiences.

Importantly, previous research also provides some indirect evidence for the existence of such within-person associations between psychological need satisfaction and sleep among university students. For instance, expecting to take a test the next day, which may lead to pressured studying and performance anxiety, thereby giving rise to experiences of autonomy and competence frustration, has been shown to relate to shorter daily sleep duration (Galambos et al. 2009). A few studies also indicate that socializing with friends and social support, both of which presumably engender relatedness satisfaction, relate to within-person fluctuations in sleep. For example, socializing with friends has been shown to relate to higher daily and monthly quality of sleep (Galambos et al. 2009, 2010), whereas social support related to higher yearly quantity of sleep (Galambos et al. 2013). Diary studies have also shown a link between daily need satisfaction and more daily positive affect and less negative affect (e.g., Ryan et al. 2010), both of which have previously been linked to within-person fluctuations in university students' quality and quantity of sleep (Galambos et al. 2009, 2010). However, no studies have used direct and SDT-based measures of the psychological needs to examine within-person associations between need satisfaction and sleep among university students.

\section{The role of stress}

Acknowledging that the association between need satisfaction and sleep is likely bidirectional, the question arises why need-based experiences might relate to individuals' quality and quantity of sleep? In other words: how can one part of the presumed bidirectional process be explained? Researchers have theorized that stressful events evoke powerful feelings of threat and arousal which likely obstruct restful sleep at night (Dahl and Lewin 2002). Accordingly, various studies indicate that perceived stress is detrimental to sleep among university students. For example, a crosssectional study in a large sample of university students found perceived stress to be associated with poorer sleep quality (Lund et al. 2010). Furthermore, a study of first year university students involving seven assessments throughout the year found sleep quality and sleep quantity to be lower in months when stress was higher (Galambos et al. 2010). Similarly, another longitudinal study found sleep and stress to co-vary across years with students reporting longer sleep duration and fewer sleep disturbances during less stressful years (Galambos et al. 2013). Further, increases in perceived stress during potentially stressful periods, such as exam periods, have been shown to be predictive of diminished sleep quality (Zunhammer et al. 2014).

Exam periods not only involve increased stress and more sleep difficulties (e.g., Zunhammer et al. 2014), but are also likely to result in reduced need satisfaction. Exams are a time when students are likely to feel restricted in their freedom, have doubts about their capabilities to master the study material and achieve desired grades, and have more limited social interactions due to high academic pressure and demands. Thus, lower need satisfaction during exam 
periods may co-vary with symptoms of stress such as tension, over-arousal or inability to relax, which in turn would likely impair daytime functioning and impede restful sleep at night.

In line with this reasoning a number of previous studies have provided direct evidence for the association between need-based experiences and indicators of stress (see Weinstein and Ryan 2011 for an overview). For example, in a study of dancers higher psychological need satisfaction was associated with lower stress response during performance conditions (Quested et al. 2011). In addition, an experimental study showed that participants who engaged in daily need satisfying activities reported reduced stress following the 1 week intervention period (Weinstein et al. 2016). Furthermore, previous research has also provided some evidence for the intervening role of stress in the relation between need-based experiences and sleep. For example, a diary study found that fluctuations in participants' experiences of need frustration from day to day co-varied with symptoms of stress, which in turn related to fluctuations in both quality and quantity of sleep and subjective energy (Campbell et al. 2018). In addition, a prospective cross-sectional study among individuals with unexplained chronic fatigue found that individuals' who experienced more need frustration during the past week, also reported higher symptoms of stress. Higher symptoms of stress, in turn, were associated with experiencing more negative sleep-related thoughts, which in turn, related to poorer sleep quality- and quantity during a stay at sleep laboratory (Campbell et al. 2017).

\section{The present research}

In the present short-term longitudinal study we assessed university students' need satisfaction and their quality and quantity of sleep before, during and after an exam period. To be consistent with the Pittsburgh Sleep Quality Index (PSQI; Buysse et al. 1989), one of the most commonly used measures to assess sleep, we also included various indicators of daytime dysfunction (i.e., the General Vitality Scale, Ryan and Frederick 1997, and the lassitude subscale of the Inventory of Depression and Anxiety Symptoms (IDAS; Watson et al. 2007)), which tapped into feelings of energy and exhaustion. Although such measures do not directly assess sleep, we deemed their inclusion important given their obvious relation with sleep.

First, we examined how psychological need experiences, sleep and daytime functioning unfolded as participants went through an exam period. We chose to assess university students during an exam period because students are likely to show substantial change and variability in sleep during exams (Zunhammer et al. 2014). We expected negative outcomes (i.e., stress and poor sleep quality) to increase from the pre- to the exam period and to decrease from the exam to the post-exam period, while the reverse pattern was expected for positive outcomes (i.e., need satisfaction and sleep quantity; Hypothesis 1). Second, apart from inspecting these mean-level changes we also examined the covariation between changes in need satisfaction and changes in the sleep and daytime outcomes across time, thereby examining whether they increased and decreased in tandem. We expected decreases in need satisfaction to go along with increases in negative outcomes and decreases in positive outcomes (Hypothesis 2). Finally, we considered the intervening role of stress in the relation between need satisfaction and the outcomes. That is, we tested an integrated model that posits within-person changes in stress as an intervening variable in the relation between changes in need satisfaction and changes in the sleep and daytime outcomes (Hypothesis 3).

\section{Method}

\section{Participants and procedure}

All participants were recruited at the host University through an electronic learning platform for students. Upon recruitment all participants were informed that they would be required to complete an online questionnaire at three different time points; once in the month of May, once in the month of June during an examination period and once in the month of July. The link to the questionnaire was sent at the beginning of each month and participants were asked to complete the questionnaire on a Sunday or at the very latest a Monday morning with respect to the preceding week. In contrast to the first and third assessments, during the second assessment participants were given specific instructions to complete the questionnaire at the end of the week in which they had the highest number of exams. The average number of exams during the exam period was 5.84 ; $S D=2.39$; range $2-13$. The average number of days between assessments was 24.34; $S D=6.92$ between the 1 st and 2 nd and 38.01 ; $S D=7.31$ between the 2 nd and $3 \mathrm{rd}$. Text messages were sent throughout the duration of the study to remind the participants to fill in the questionnaire if they had not previously done so. All participants were assigned a unique code to ensure confidentiality and that the data from each participant could be matched across the three waves. The first page of the online questionnaire stipulated the voluntary and confidential nature of the study. All participants were required to read this page before providing online informed consent, which was received from all participants. The study was conducted according to the ethical rules presented in the General Ethical Protocol of the Faculty of Psychology and Educational Sciences of the host University. Given that all participants were over 18 years of age and filled in an 
online informed consent form in which they were informed that they could withdraw from the study at any point and that their anonymity was guaranteed, formal ethical approval was not required.

Given that (a) this study was fairly unique in terms of the design used and the analyses conducted and that (b) equivalent previous studies are considered necessary to conduct a power analysis (Levine and Ensom 2001), we were unable to rely on effect sizes from prior studies to perform an a priori power analysis. The final sample consisted of 121 Belgian emerging adults ( $78 \%$ female) with a mean age of 21.69 years (ranging from 19 to $25 ; S D=1.39$ ). Eighty-six percent of the participants were university students and the remaining $14 \%$ were following a non-university form of higher education. Of the 121 respondents, 87 (72\%) participated in all three waves. Sample attrition was examined in two steps. First, individuals who participated in all three waves were dummy coded as 1 (retention), and individuals who participated in only one or two waves were coded as 0 (drop-out). Logistic regression analysis was then performed by entering demographic variables (e.g., age and gender) in Step 1 and all Time 1 study variables in Step 2 to predict sample attrition. Model Chi square for Step $1 \chi^{2}(2)=5.12$, $p=0.08$ and Step $2 \chi^{2}(5)=7.87, p=0.16$ was not significant, indicating that the demographic and study variables did not contribute significantly to the prediction of dropout. Furthermore, Little's MCAR was non-significant ( $p=0.07$; $\chi^{2} / \mathrm{df}=185.91 / 159$; normed $\chi^{2}$ of 1.17), indicating that data were likely to be missing completely at random. As a result, full information maximum likelihood (FIML) was used to handle missing data in SEM (Little and Rubin 1987).

\section{Measures}

\section{Basic psychological needs}

The satisfaction of the needs for autonomy, competence, and relatedness was assessed using the Basic Psychological Need Satisfaction and Need Frustration Scale (BPNSNFS; Chen et al. 2015). The scale consists of 24 items, 8 items per need, 4 of which tap into need satisfaction and 4 of which tap into need frustration. Participants rated all items on a scale of 1 (not at all true) to 5 (very true) with respect to the preceding week. Because changes in the mean scores of the 12 items assessing need satisfaction and the 12 items assessing need frustration were highly correlated across measurement moments (i.e., preexam to exam $r=-0.82, p<0.001$; exam to post-exam $r=-0.86, p<0.001$ ), we used a composite score of need satisfaction in all subsequent analyses. This was done by reverse scoring the 12 items assessing need frustration and then creating three separate need scores by averaging the 8 items assessing autonomy $(\alpha=0.79-0.84)$, competence
( $\alpha=0.87-0.92)$, and relatedness $(\alpha=0.85-0.91)$. Consistent with previous research a composite score of need satisfaction ( $\alpha=0.91-0.93$ ) was then created by averaging the sum of the three need variables (Campbell et al. 2015).

\section{Stress}

Symptoms of stress were assessed using the stress subscale from the short-form version of the Depression Anxiety and Stress Scale (DASS; Lovibond and Lovibond 2004; Campbell et al. 2017). The stress subscale consists of 7 items which measure the prevalence of symptoms of stress during the past week (e.g., "I tended to over-react to situations"). Participants rated items on a 4-point Likert scale, ranging from 0 (did not apply to them) to 3 (applied very much, or most of the time). Cronbach's alpha ranged between 0.89 and 0.92 .

Sleep and day-related measures Several measures were included to assess quality and quantity of sleep and daytime functioning. All measures were adjusted to ensure that they assessed the same timeframe (i.e., the past week). A principal component analysis (PCA; See preliminary analysis) was performed to examine the structure underlying the assessed sleep parameters. Below the indicators of the three retained components are described.

Poor sleep quality Poor sleep quality during the previous week was assessed using six indicators. These were the subjective poor sleep quality, sleep latency, sleep disturbances and use of sleep medication component scores from the PSQI (Buysse et al. 1989; Campbell et al. 2015), the insomnia subscale from the IDAS (Watson et al. 2007; e.g., "During the past week I woke up frequently throughout the night"; 6 items), and a composite of 2 items assessing sleep disturbing cognitions (Campbell et al. 2015; e.g., "Worrying" and "Somber thoughts"; 2 items). The insomnia items ( $\alpha$ range $0.82-0.89$ ) were rated on a 5-point Likert scale ranging from 1 (not at all) to 5 (very much so) and the sleep disturbing cognitions ( $\alpha$ range $0.78-0.82$ ) were rated on an event-frequency scale ranging from 0 (Not experienced during the past week) to 3 (Experienced three or more times).

Sleep quantity The previous week's sleep quantity was assessed using the sleep duration and habitual sleep efficiency component scores from the PSQI (Buysse et al. 1989). Both scores were reversed such that a higher score was representative of longer sleep duration and higher sleep efficiency.

Daytime dysfunction Daytime dysfunction was assessed using the daytime dysfunction component score from the PSQI (Buysse et al. 1989), a measure of subjective vitality 
(General Vitality Scale; Ryan and Frederick 1997; e.g., "I felt energized"; 7 items), and the lassitude subscale from the IDAS (Watson et al. 2007; e.g., "During the past week I felt sleepy and drowsy"; 6 items). The vitality ( $\alpha$ range 0.84 0.86 ) and lassitude items ( $\alpha$ range $0.78-0.82$ ) were rated on a 5-point Likert scale ranging from 1 (completely disagree or not at all) to 5 (completely agree or very much so).

\section{Statistical analysis}

To examine the relation between intra-individual changes in need experiences and sleep and daytime parameters across measurement moments latent change models (LCMs) were tested using Mplus7 with Maximum Likelihood as estimator. LCMs estimate within-person change across two adjacent waves, using latent variables for intercept (i.e., level) and slope (i.e., change over time) (Beyers and Goossens 2008). Variance in the slope indicates between-person differences in within-person change over time. Using LCMs we estimated in separate models change from the pre-exam period to the exam period and from the exam period to the postexam period. The decision to model change across the three waves in two separate models is informed by the fact that the nature of the transition from Wave 1 to Wave 2 (i.e., transitioning into an exam period) is qualitatively different from the nature of the transition from Wave 2 to Wave 3 (i.e., transitioning out of the exam period). Given that both transitions are qualitatively different it seemed less appropriate to model change across the three waves using one overall parameter of change.

Each latent change model consisted of a longitudinal measurement model defining the latent variables (i.e., need satisfaction, sleep quality, sleep quantity, daytime dysfunction and stress) at each time point by their respective indicators and a structural model which defined latent level and change factors for each latent variable and further specified how these levels and changes were interrelated (Hertzog et al. 2003). Further, co-variances among the residuals of the same indicators over time were specified (Sörbom 1975) and background variables (i.e., age and gender) were controlled for in all models. Model fit was evaluated using the comparative fit index (CFI); the root squared error of approximation (RMSEA) and the Standardized Root Means Square Residual (SRMR). An acceptable fit was indicated by CFI values of 0.90 or above, and RMSEA and SRMR values of around 0.08 or below (Hu and Bentler 1999; Kline 2005).

In the longitudinal measurement model, each latent variable was represented by two parcels. Parcels were created by combining stronger loading items with weaker loading items from each scale (Little et al. 2002). Need satisfaction was indicated by the same two six-item parcels at each time point whereas stress was indicated by one three-item and one four-item parcel. Indicators for the latent constructs of sleep quality, sleep quantity and daytime dysfunction were determined from a second order PCA (see preliminary analyses) performed at all three waves, thereby using scale scores rather than items as indicators and averaging the standardized factor loadings across the three waves. As more than two indicators were retained for sleep quality and daytime dysfunction, stronger and weaker loading factor scores were combined to create two parcels, whereas sleep quantity was represented by the two indicators determined in the PCA.

\section{Results}

\section{Preliminary analyses}

\section{Factor structure}

The structure underlying the battery of sleep parameters assessed at each time point was examined using PCA with promax rotation. Promax rotation was chosen because the underlying sleep and daytime parameters were assumed to be correlated. At each wave PCA resulted in three components with eigenvalues greater than 1 , which combined explained $63.31 \%, 63.29 \%$ and $65.34 \%$ of the variance at Waves $1-3$, respectively. Inspection of the scree plot also indicated a three factor solution at each wave. Standardized loadings averaged across the three waves varied between 0.41 and 0.87 , with an average of 0.62 . The retained components were similar to those found in Campbell et al. (2015) and clearly represented poor sleep quality (including sleep latency, insomnia symptoms, sleep disturbing cognitions, sleep quality, use of sleep medication and sleep disturbances), sleep quantity (including habitual sleep efficiency and sleep duration) and daytime dysfunction (including daytime dysfunction, lassitude, and vitality).

\section{Descriptive statistics and correlations}

Composite scores were created for sleep quantity, poor sleep quality and daytime dysfunction by computing the mean of the corresponding indicators determined in the PCA. Correlations between all study variables across the three measurement waves are available in Table 1 in the supplementary file. All study variables were related in the expected directions except for sleep quantity which was unrelated to need satisfaction at $\mathrm{T} 2 .{ }^{1}$ All rank-order stability coefficients

\footnotetext{
1 As shown in Table 2 in the supplementary file all three separate needs (i.e., for autonomy, competence and relatedness) displayed similar relations with the study variables. Furthermore, repeated measures ANOVA's revealed that each of the three needs displayed a significant quadratic trend across the three time points [autonomy: $F(1,86)=89.02, p<.001, \eta^{2}=0.51 ;$ competence: $F(1,86)=45.48$, $p<.001, \eta^{2}=0.35$; relatedness: $\left.F(1,86)=8.62, p=.004, \eta^{2}=0.09\right]$.
} 
Table 1 Parameter estimates and fit indices of the univariate latent change models

\begin{tabular}{|c|c|c|c|c|c|c|c|c|c|c|c|}
\hline \multirow[t]{3}{*}{ Variable } & \multicolumn{11}{|c|}{ Parameter estimates } \\
\hline & \multicolumn{2}{|l|}{ Level } & \multicolumn{3}{|c|}{ Change pre-exam to exam period } & \multicolumn{3}{|c|}{ Change exam to post exam period } & \multicolumn{3}{|c|}{ Fit indices } \\
\hline & $M$ & $\mathrm{~s}^{2}$ & $M$ & $\mathrm{~s}^{2}$ & Range & $M$ & $\mathrm{~s}^{2}$ & Range & RMSEA & $\mathrm{CFI}$ & $\overline{\text { SRMR }}$ \\
\hline Need satisfaction & $3.47 * * *$ & $0.24 * * *$ & $-\mathbf{0 . 2 0} * * *$ & $0.19 * * *$ & -1.35 to 0.72 & $\mathbf{0 . 4 2} * * *$ & $0.21 * * *$ & -0.38 to 1.73 & 0.00 & 1 & 0.09 \\
\hline Stress & $\mathbf{0 . 8 4} * * *$ & $\mathbf{0 . 4 2} * * *$ & $0.23 * * *$ & $0.31 * * *$ & -0.74 to 1.27 & $-0.66 * * *$ & $\mathbf{0 . 3 8} * * *$ & -2.44 to 0.13 & 0.09 & 0.98 & 0.04 \\
\hline Daytime dysfunction & 0.01 & $0.64 * *$ & $0.59 * * *$ & $-0.79 * * *$ & -0.91 to 2.17 & $-0.79 * * *$ & $1.01 * * *$ & -3.35 to 0.65 & 0.04 & 0.99 & 0.04 \\
\hline Sleep quantity & 3.51*** & $\mathbf{0 . 2 1} * * *$ & -0.09 & 0.13 & -0.77 to 0.64 & $0.16^{*}$ & $0.36 * *$ & -1.25 to 1.19 & 0.00 & 1 & 0.12 \\
\hline Poor sleep quality & 0.00 & $0.21 * * *$ & $0.19 * * *$ & $\mathbf{0 . 0 8}^{* *}$ & -0.17 to 0.65 & $-0.25 * * *$ & $0.07 * *$ & -0.84 to 0.05 & 0.00 & 1 & 0.03 \\
\hline
\end{tabular}

Indicators of poor sleep quality and daytime dysfunction use standardized scores. Estimates in bold remained significant after correcting for multiple comparisons (Bonferroni correction), $\mathrm{p}<0.01$

$R M S E A$ root mean square error of approximation, CFI comparative fit index, SRMR standardized root mean square residual $* p<0.05 ; * * p<0.01 ; * * *<<0.001$

Table 2 Correlations between level and change parameters from multivariate latent change models

\begin{tabular}{|c|c|c|c|}
\hline & \multicolumn{3}{|c|}{ Need satisfaction } \\
\hline & Level & Change12 & Change23 \\
\hline \multicolumn{4}{|c|}{ Poor sleep quality } \\
\hline Level & $-0.47 * * *$ & 0.04 & -0.01 \\
\hline Change 12 & $0.34 *$ & $-0.25^{*}$ & -0.03 \\
\hline Change 23 & -0.14 & $0.31 * *$ & $-0.35^{*}$ \\
\hline \multicolumn{4}{|c|}{ Sleep quantity } \\
\hline Level & $0.44 * * *$ & -0.12 & -0.11 \\
\hline Change12 & $-0.39 *$ & $0.36^{*}$ & 0.05 \\
\hline Change 23 & 0.20 & $-0.21^{*}$ & -0.08 \\
\hline \multicolumn{4}{|c|}{ Daytime dysfunction } \\
\hline Level & $-0.77 * * *$ & $0.25 *$ & 0.22 \\
\hline Change12 & $0.23 *$ & $-\mathbf{0 . 6 0} * * *$ & $0.32 * *$ \\
\hline Change23 & $0.26^{*}$ & $0.21 *$ & $-0.63 * * *$ \\
\hline \multicolumn{4}{|l|}{ Stress } \\
\hline Level & $-0.57 * * *$ & 0.08 & $0.28 *$ \\
\hline Change12 & 0.04 & $-\mathbf{0 . 5 0} * * *$ & 0.13 \\
\hline Change23 & $0.26^{*}$ & $0.30 * *$ & $-0.49 * * *$ \\
\hline
\end{tabular}

Change12 refers to changes from the pre exam to the exam period. Change 23 refers to changes from the exam to the post exam period. Correlations in bold remained significant after correcting for multiple comparisons (Bonferroni correction), $p<0.001$

$* p<0.05 ; * * p<0.01 ; * * * p<0.001$

were significant ranging from 0.26 to 0.65 apart from two exceptions, namely sleep quantity at $\mathrm{T} 2$ and $\mathrm{T} 3$ and daytime dysfunction at $\mathrm{T} 2$ and $\mathrm{T} 3$.

For descriptive purposes the seven components of the PSQI were summed to compute a Global PSQI score at each time point. A cut-off of $>5$ is used to distinguish between "good sleepers" and "poor sleepers", with higher scores representing poorer global sleep quality. (Buysse et al. 1989). A repeated measures ANOVA revealed a significant quadratic trend in Global PSQI scores across the three time points, $F(1,86)=44.31, p<0.001, \eta^{2}=0.34$. Post-hoc tests using the Bonferonni correction revealed that Global PSQI scores were significantly higher at $\mathrm{T} 2(M=5.70, \mathrm{SD}=2.45)$ than at $\mathrm{T} 1(M=4.13, \mathrm{SD}=2.70)$ and $\mathrm{T} 3(M=3.92, \mathrm{SD}=2.35)$, whereas differences between $\mathrm{T} 1$ and $\mathrm{T} 3$ were not significant. The percentage of individuals with a Global PSQI score $>5$ displayed a similar pattern and was highest at $\mathrm{T} 2=38 \%$ relative to $\mathrm{T} 1=30.1 \%$ and $\mathrm{T} 3=17 \%$.

\section{Background variables}

The relation between participants' background characteristics (i.e., age and gender) and the study variables was examined using a MANCOVA with gender as a between-subjects variable, age as a covariate and all the study variables as dependent variables. Participants' age, $F(9,47)=0.89$, $p=0.56$, did not yield a significant multivariate main effect, whereas gender did, $F(9,47)=2.58, p=0.01, \eta^{2}=0.33$, with males reporting significantly longer sleep quantity than females during the exam period, $F(1,55)=2.62, p=0.01$, $M_{\text {men }}=0.85, M_{\text {women }}=0.31$.

\section{Primary analyses}

\section{Hypothesis 1: examining mean-level change}

Univariate LCMs were estimated for all study variables. Table 1 provides an overview of the parameter estimates and fit indices for each model. On average, significant mean level change was found for all study variables from the preto exam period and from the exam to the post- exam period with one exception, namely sleep quantity for which no significant mean-level change was found from pre- to exam period. All negative outcomes (i.e., stress, daytime dysfunction and poor sleep quality) displayed an inverted U-shaped pattern whereas all positive outcomes (i.e., need satisfaction 
Fig. 1 Mean-level changes in stress across time-points. Note The error bars depicted in the figure represent the variance (s2) of the sample

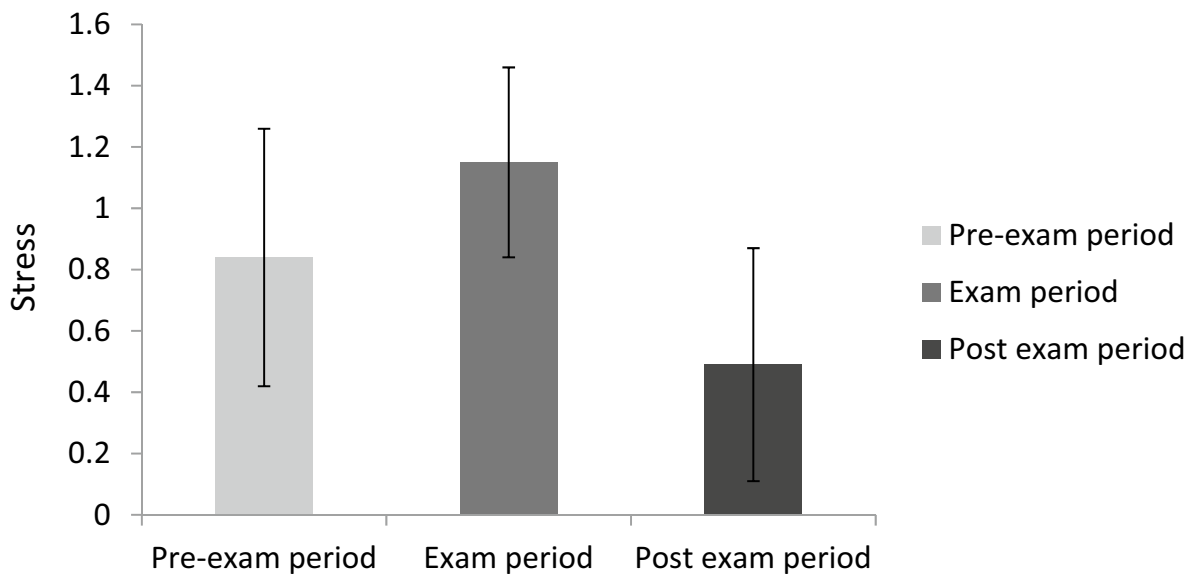

and sleep quantity) displayed a U-shaped pattern. In other words, on average, as participants were exposed to an exam period, their experiences of subjective stress, daytime dysfunction and poor sleep quality increased, while their experiences of need satisfaction decreased. Furthermore, once the exam period was over, participants showed improvements in need satisfaction, stress and sleep outcomes (see Fig. 1 for an illustration).

\section{Hypothesis 2: correlated changes in need satisfaction, stress and sleep outcomes}

There were four outcome variables (i.e., poor sleep quality, sleep quantity, daytime dysfunction, and stress), resulting in four multivariate LCM's. Each model included both the level and change factors for the need composite and one outcome, allowing for the examination of correlations between the levels and the correlated change in need satisfaction and the four outcomes. All models fitted the data adequately with the average fit being RMSEA $=0.05, \mathrm{CFI}=0.98$ and $\mathrm{SRMR}=0.09$ and the poorest fit being $\mathrm{RMSEA}=0.08$, $\mathrm{CFI}=0.97$ and $\mathrm{SRMR}=0.09$. As shown in Table 2, the intercepts of need satisfaction and the four outcomes were significantly interrelated in the expected direction. More importantly, as hypothesized, the changes were interrelated suggesting that the changes in need satisfaction and the outcomes occurred in tandem. For example, changes in both need satisfaction and poor sleep quality were negatively related indicating that as need satisfaction decreased from the pre- to the exam period sleep quality deteriorated and that as need satisfaction increased from the exam- to the post-exam period sleep quality also improved. ${ }^{2}$

\footnotetext{
${ }^{2}$ As shown in Table 2 some caution is needed when interpreting the covariation between need satisfaction and both sleep quality (across both transitions) and sleep quantity (from the pre- to the exam period) because they became non-significant after correcting for multiple testing. As such, these findings are in need of replication.
}

\section{Hypothesis 3: testing the proposed integrated model}

Latent variable scores of levels and changes calculated from the previous multivariate LCMs were used to examine whether the relationship between changes in need satisfaction and changes in sleep and daytime dysfunction were accounted for by changes in stress. This set of analyses allowed us to examine whether, for example, a decrease in need satisfaction from pre- to exam-period would relate to increases in poor sleep quality via increases in perceived stress. To test for the intervening role of stress, we estimated a model including direct effects from need satisfaction to the outcomes and indirect effects via stress. The final results of these models are shown in Fig. 2a (i.e., sleep quality), b (i.e., sleep quantity), c (i.e., daytime dysfunction). In each figure the first coefficients reported represent changes from the pre- exam to the exam period and the second coefficients represent changes from the exam to the post exam period.

Given that these models were saturated, therefore resulting in perfectly fitting models, our primary interest was in the direct and indirect associations between the study variables. With respect to poor sleep quality (i.e., Fig. 2a), the association with changes in need satisfaction was fully accounted for by changes in stress across both transitions. To illustrate, the decrease in need satisfaction from the preto the exam period co-varied with an increase in poor sleep quality which could be explained by the increase in experienced stress during this transition (as reflected by the first coefficients reported, that is., -0.44 and 0.23 ). With regard to changes in daytime dysfunction, the associations with changes in need experiences were partially accounted for by changes in stress across all changes. In other words, the increase in stress from the pre- to the exam period and the subsequent decrease from the exam to the post-exam period partially explained why changes in need satisfaction related to changes in daytime dysfunction during these transitions. Finally, with regard to changes in sleep quantity, across both transitions changes in need satisfaction related to changes 

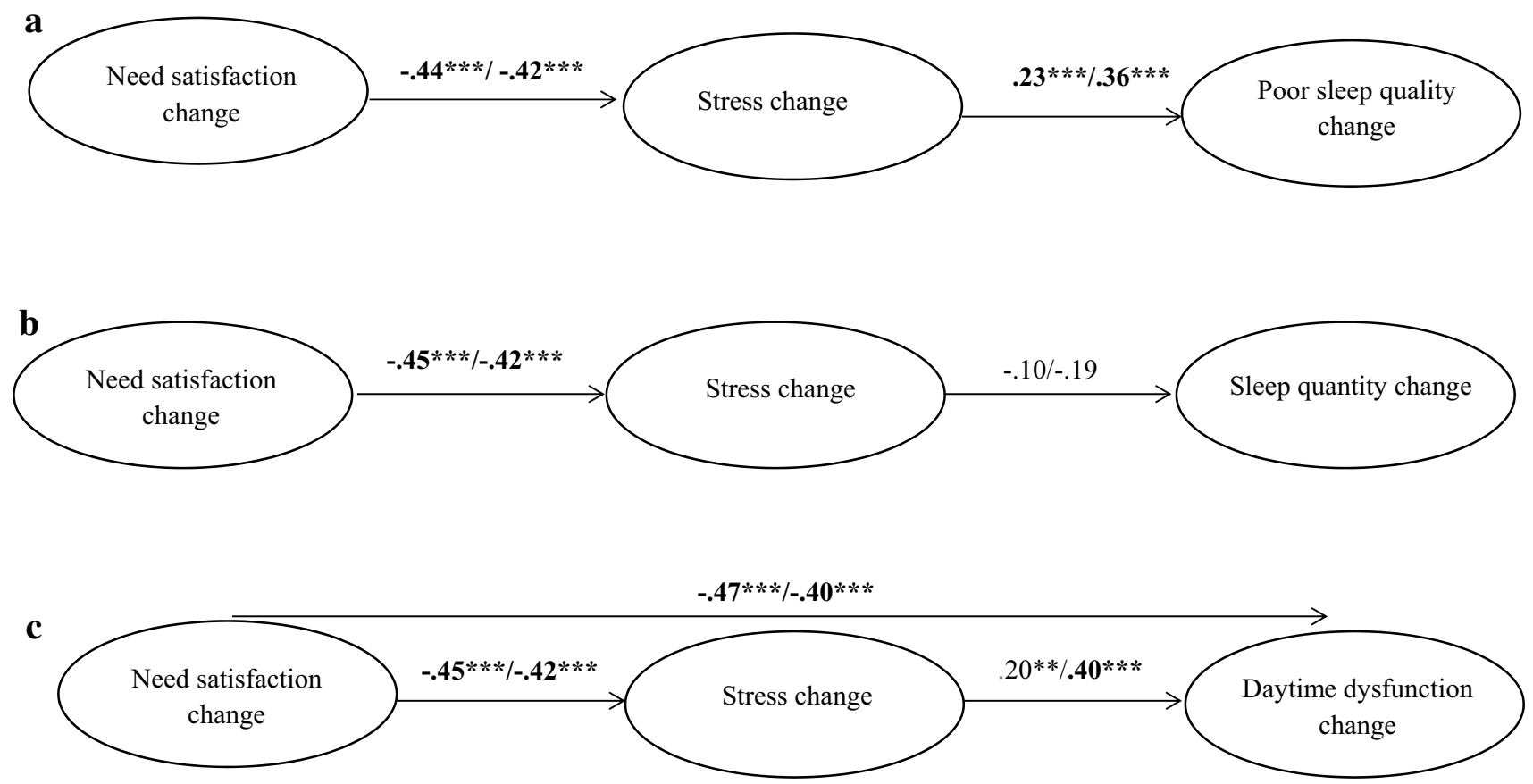

Fig. 2 a Changes in psychological need satisfaction predicting changes in poor sleep quality via changes in stress. b Changes in psychological need satisfaction predicting changes in sleep quantity via changes in stress. $\mathbf{c}$ Changes in psychological need satisfaction predicting changes in daytime dysfunction via changes in stress.

in stress, however changes in stress, in turn, were unrelated to changes in sleep quantity. The indirect effects between changes in need satisfaction and changes in both sleep quality and daytime dysfunction via changes in stress were significant across all transitions [range CI 95\% (-.24; 0.10)].

\section{Discussion}

Consistent with previous research (e.g., Galambos et al. 2009, 2010, 2013), the present study found sleep in emerging adulthood and, more specifically in a sample of university students, to vary considerably within individuals. As pointed out by Galambos et al. (2009) such within-person fluctuations beg for explanation. That is, there is a need to identify predictors with equal variability that evolve in tandem with emerging adults' quality and quantity of sleep. In the present study we aimed to extend previous findings by considering psychological predictors of university students' sleep from an established psychological framework during a particularly relevant and potentially stressful period. Specifically, we investigated (a) the covariation between changes in the satisfaction of one's basic psychological needs, as conceived within SDT (Deci and Ryan 2000), and changes in emerging adults' day and sleep-related functioning and (b) the intervening role of changes in stress in these associations.
$* p<0.05 ; * * p<0.01 ; * * * p<0.001$. The first coefficients reported represent changes from the pre- to the exam period and the second coefficients represent changes from the exam to the post exam period. Coefficients in bold remained significant after correcting for multiple comparisons (Bonferroni correction), $\mathrm{p}<0.008$

Meaningful mean-level changes were observed as university students prepared for, were exposed to and emerged from an exam period. During the exam period participants reported increases in negative outcomes (i.e., poor sleep quality and daytime dysfunction) and decreases in positive outcomes (i.e., need satisfaction). As participants emerged from the exam period the reverse pattern of findings was observed (i.e., decreases in negative outcomes and increases in positive outcomes). In other words, both need-based and sleep-related functioning deteriorated as participants moved from the pre- to the exam period, and returned to and even went beyond the initial levels once the exam period was over. Overall, these mean-level changes in the outcomes were in line with our expectations and are indicative of the dynamic nature of the variables under investigation.

Second, we then examined whether within-person changes in psychological need satisfaction were associated with within-person changes in the day and sleep outcomes across time. This appeared to be the case. On average, as university students went through an exam period, their sleep and daytime functioning fluctuated in parallel with their need-based experiences such that decreases in need satisfying experiences went hand in hand with poorer sleep quality, shorter sleep quantity and worse daytime functioning. Moreover, as university students emerged from the exam period, an improvement in their need satisfaction was accompanied 
by a rise in their sleep quality as well as more adaptive daytime functioning. Presumably, the exposure to need-thwarting experiences during exam periods erodes energy levels and elicits more cognitive and somatic arousal, which in turn likely requires more emotional processing which then interferes with sleep at night.

Finally, the assessment of stress allowed us to examine whether changes in stress would account for the relation between changes in need satisfaction and changes in the outcomes. In general, the rise and fall in stress as university students moved in and out of the exam period helped to explain why shifts in need satisfaction related to shifts in both the day and sleep-related outcomes. While the association between stress and poor sleep has already been demonstrated in various previous studies (e.g., Galambos et al. 2010, 2013; Zunhammer et al. 2014), the present results build on previous findings by suggesting that changes in psychological need satisfaction may contribute to changes in stress when exposed to a potentially stressful event. Hence, the present findings may help to identify specific experiences which lead to an event, such as an exam period, to be appraised by students as threatening thereby possibly engendering symptoms of stress and subsequent sleep disturbances. Moreover, the identification of need experiences as potential sources of stress may inform interventions which seek to help university students to reduce stress by providing specific experiences which can be targeted through intervention (e.g., Weinstein et al. 2016).

Although these findings provide some preliminary evidence for the intervening role of stress, two findings should be highlighted. First, in the integrated models, need experiences continued to yield a direct association with daytime dysfunction, suggesting that other explanatory variables, apart from stress, should be considered. One primary candidate is mindfulness (Brown and Ryan 2003). Need frustrating experiences likely erode available energy resulting in a more inward looking attitude and a decreased awareness of what is occurring in the present moment. This decreased awareness, or lack of a mindful approach, may in turn partly explain why need frustrating experiences may translate into poorer daytime functioning or even poorer quality sleep at night (see Campbell et al. 2015; Hulsheger et al. 2014). Second, although decreased need satisfaction was found to co-vary with shorter sleep quantity from the pre- to the exam period, in the integrated models changes in stress were unrelated to changes in sleep quantity. Perhaps university students respond to decreased need satisfaction during exam periods by engaging in compensatory behaviors that they hope will provide some need fulfillment like for example, spending excessive time on social media, which in turn may lead to shorter sleep duration. Future studies could assess other self-regulatory behaviors, such as sleep hygiene, in order to obtain a better understanding of how decreased need satisfaction may obstruct sleep duration.

Another possibility is that poor sleep quantity and quality may also reciprocally impact on university students' need-based experiences. Indeed, it is important to note that these findings need to be interpreted with caution given the correlational nature of the data. Future experimental research is needed to determine the exact direction of effects between need satisfaction, stress and sleep disturbance. In fact, a recent experimental study (Campbell et al. in pressc), involving partial sleep deprivation indicated that reduced need satisfaction also follows from reduced sleep quantity. Specifically, participants in an experimental group, who restricted their sleep to $5 \mathrm{~h}$ a night for three consecutive nights, reported reduced need satisfaction after 3 days of sleep deprivation, relative to a control group, who slept as usual throughout the 3 -day period. Thus, the longitudinal associations obtained in the current study most likely reflect ongoing bidirectional dynamics, with sleep not only being an outcome of psychological need-based experiences and stress but also affecting these psychological experiences.

\section{Practical implications}

The present findings indicate that during exam periods students are likely to experience reduced satisfaction of their basic psychological needs, which in turn is likely to be accompanied by increases in stress and sleep disturbances. These findings emphasize the dynamic and changeable nature of need-based experiences and suggest that psychological needs may be especially susceptible to intervention. In line with this, a 1 week intervention study which sought to help individuals highly vulnerable to distress (i.e., Syrian refugees) identify and participate in daily need-satisfying activities demonstrated that participants reported significant decreases in stress post-intervention (Weinstein et al. 2016). Hence, short-term interventions which encourage students to engage in small, manageable daily activities which satisfy basic psychological needs may help to reduce stress and sleep difficulties during exam periods. In addition to encouraging engagement in need satisfying activities, students could also be helped to become more aware of and less reactive to experiences of need frustration. This could be achieved for example, by encouraging students to adopt techniques which facilitate accepting, non-judgmental present moment awareness (i.e., mindfulness), such as meditation practice. Furthermore, given the possible reciprocity between need-based experiences and sleep, students could also be encouraged to maintain healthy sleep hygiene behaviors (i.e., maintain a regular sleep schedule and refrain from drinking caffeinated beverages late at night) during exam periods, so as to try and maintain their need satisfaction. 


\section{Limitations and suggestions for future research}

This study had several limitations that can be overcome in future research. The main limitation of the present study is that the analyses performed do not allow for conclusions about the direction of effects. For example, poor sleep during exam periods may not only result from, but may also contribute to stress, which in turn may lead to reduced need satisfaction. Experimental work involving a random exposure to need satisfying or need frustrating experiences is needed to allow for causal conclusions to be drawn about whether decreased need satisfaction really drives the maladaptive pattern of increased stress and poor sleep quality. In addition, all measures were self-reported which can inflate observed associations due to shared method variance. Moreover, reliance on self-reports may have undermined validity for certain measures such as sleep duration. These limitations could be overcome by using objective measures such as actigraph watches to assess sleep in future studies. Furthermore, as our sample was fairly homogenous and consisted mainly of females, future studies should examine whether these findings generalize to emerging adults from a more diverse sociodemographic background. Another limitation of this study is that the post-exam assessment took place during a holiday period rather than during the academic semester. This may help to explain why need-based and sleep functioning were most favorable during the postexam assessment, rather than during the pre-exam assessment, the latter of which took place during the academic semester when academic demands were likely increasing. Further, given the heterogeneity in the covariation between psychological needs and sleep, future studies could examine potential moderators of these associations. For example, future studies could explore the moderating role of individuals' dispositional mindfulness (Brown and Ryan 2003) or self-critical perfectionism (Blatt 2004) as both are likely to influence reactivity to need experiences. Finally, given our findings future research could also examine whether a short-term intervention which aims to foster need satisfaction, would help students to be more resilient to stress and sleep disturbances during exam periods.

\section{Conclusion}

In sum, the present study underscores the dynamic interplay between university students' need-based experiences, daytime functioning and quality and quantity of sleep during an exam period. The findings indicate that during weeks in which university students feel pressured and ineffective in their activities and disconnected from close others, their daytime functioning and sleep is likely to be impaired. Furthermore, subjective stress was found to partially account for the relation between need satisfaction and the day and sleep outcomes, indicating that stress may play an explanatory role in these associations. These findings suggest that university students should be helped to recognize need frustrating experiences and taught skills to minimize their impact by, for example, adopting a more mindful approach or, alternatively, students should be encouraged to pay attention to their sleep hygiene during exam periods, given that lowered need satisfaction may also stem from poor sleep.

Funding This study was funded by the Fund for Scientific Research Flanders (FWO.OPR.2013. 0140.01 - IV2).

\section{Compliance with ethical standards}

Conflict of interest The authors declare that they have no conflict of interest.

\section{References}

Ahrberg, K., Dresler, M., Niedermaier, S., Steiger, A., \& Genzel, L. (2012). The interaction between sleep quality and academic performance. Journal of Psychiatric Research, 46, 1618-1622. https ://doi.org/10.1016/j.jpsychires.2012.09.008.

Arnett, J. J. (2000). Emerging Adultho:od: A theory of development from the late teens through the twenties. American Psychologist, 55, 469-480. https://doi.org/10.1037//0003-066X.55.5.469.

Beyers, W., \& Goossens, L. (2008). Dynamics of perceived parenting and identity formation in late adolescence. Journal of Adolescence, 31, 165-184. https://doi.org/10.1016/j.adolescenc e.2007.04.003.

Blanco, C., Okuda, M., Wright, C., Hasin, D. S., Grant, B. F., Liu, S.-M., \& Olfson, M. (2008). Mental health of college students and their non-college-attending peers. Archives of General Psychiatry, 65, 1429-1437.

Blatt, S. J. (2004). Experiences of depression: Theoretical, clinical and research perspectives. Washinton, DC: American Psychological Association.

Brown, K. W., \& Ryan, R. M. (2003). The benefits of being present: Mindfulness and its role in psychological well-being. Journal of Personality and Social Psychology, 84, 822-848. https://doi. org/10.1037/0022-3514.84.4.822.

Buysse, D. J., Reynolds, C. F., Monk, T. H., Berman, S. R., \& Kupfer, D. J. (1989). The Pittsburgh Sleep Quality Index-A new instrument for psychiatric practice and research. Psychiatry Research, 28, 193-213. https://doi.org/10.1016/0165-1781(89)90047-4.

Campbell, R., Soenens, B., Weinstein, N., \& Vansteenkiste, M. (in press-c). The impact of partial sleep deprivation on psychological functioning: Effects on mindfulness and basic psychological need satisfaction. Mindfulness.

Campbell, R., Tobback, E., Delesie, L., Vogelaers, D., Mariman, A., \& Vansteenkiste, M. (2017). Basic psychological need experiences, fatigue and sleep in individuals with unexplained chronic fatigue. Stress and Health, 33, 645-655.

Campbell, R., Vansteenkiste, M., Delesie, L., Tobback, E., Mariman, A., Vogelaers, D., et al. (in press-b). Reciprocal associations 
between daily need-based experiences, energy, and sleep in chronic fatigue syndrome. Health Psychology.

Campbell, R., Vansteenkiste, M., Delesie, L. M., Mariman, A. N., Soenens, B., Tobback, E., Van der Kaap-Deeder, J., \& Vogelaers, D. P. (2015). Examining the role of psychological need satisfaction in sleep: A Self-Determination Theory perspective. Personality and Individual Differences, 77, 199-204. https://doi. org/10.1016/j.paid.2015.01.003.

Campbell, R., Vansteenkiste, M., Delesie, L. M., Soenens, B., Tobback, E., Vogelaers, D., \& Mariman, A. (in press-a). The role of psychological need satisfaction, sleep, and mindfulness in the health related quality of life of people living with HIV. Journal of Health Psychology.

Campbell, R., Vansteenkiste, M., Soenens, B., Vandenkerckhove, B., \& Mouratidis, A. (2018). Examining daily variation in adolescent sleep: The role of psychological need experiences. Manuscript submitted for publication.

Chen, B., Vansteenkiste, M., Beyers, W., Boone, L., Deci, E. L., Duriez, B., Lens, W., Matos, L., Mouratidis, A., Ryan, R. M., Sheldon, K. M., Soenens, B., Van Petegem, S., \& Van der KaapDeeder, J., \& Verstuyf, J. (2015). Basic psychological need satisfaction, need frustration, and need strength across four cultures. Motivation and Emotion, 39, 216-236. https://doi.org/10.1007/ s11031-014-9450-1.

Dahl, R. E., \& Lewin, D. S. (2002). Pathways to adolescent health sleep regulation and behavior. Journal of Adolescent Health, 31, 175-184. https://doi.org/10.1016/S1054-139X(02)00506-2.

Deci, E. L., \& Ryan, R. M. (2000). The 'what' and 'why' of goal pursuits: Human needs and the self determination of behavior. Psychological Inquiry, 11, 227-268. https://doi.org/10.1207/S1532 7965PLI1104_01.

Doane, L. D., Gress-Smith, J. L., \& Breitenstein, R. S. (2015). Multimethod assessments of sleep over the transition to college and the associations with depression and anxiety symptoms. Journal of Youth and Adolescence, 44, 389-404. https://doi.org/10.1007/ s10964-014-0150-7.

Galambos, N. L., Dalton, A. L., \& Maggs, J. L. (2009). Losing sleep over it: Daily variation in sleep quantity and quality in Canadian students' first semester of university. Journal of Research on Adolescence, 19, 741-761.

Galambos, N. L., Howard, A. L., \& Maggs, J. L. (2010). Rise and fall of sleep quantity and quality with student experiences across the first year of university. Journal of Research on Adolescence, 21, 342-349. https://doi.org/10.1111/j.1532-7795.2010.00679.x.

Galambos, N. L., Vargas Lascano, D. I., Howard, A. L., \& Maggs, J. L. (2013). Who sleeps best? Longitudinal patterns and covariates of change in sleep quantity, quality, and timing across four university years. Behavioral Sleep Medicine, 11, 8-22. https://doi. org/10.1080/15402002.2011.596234.

Harvey, A. G. (2008). Insomnia, psychiatric disorders, and the transdiagnostic perspective. Journal of American College Health, 17, 299-303. https://doi.org/10.1111/j.1467-8721.2008.00594.x.

Hertzog, C., Dixon, R. A., Hultsch, D. F., \& MacDonald, S. W. S. (2003). Latent change models of adult cognition: Are changes in processing speed and working memory associated with changes in episodic memory? Psychology and Aging, 18, 755-769. https ://doi.org/10.1037/0882-7974.18.4.755.

Hu, L., \& Bentler, P. M. (1999). Cutoff criteria for fit indexes in covariance structure analysis: Conventional criteria versus new alternatives. Structural Equation Modeling, 6, 1-55. https://doi. org/10.1080/10705519909540118.

Hulsheger, U. R., Lang, J. W. B., Depenbrock, F., Fehrmann, C., Zijlstra, F. R. H., \& Alberts, H. J. E. M. (2014). The power of presence: The role mindfulness at work for daily levels and change trajectories of psychological detachment and sleep quality. Journal of Applied Psychology, 99, 1113-1128. https://doi. org/10.1037/a0037702.

Kline, R. B. (2005). Principles and practice of structural equation modeling. New York: Guilford Press.

Levine, M., \& Ensom, M. H. (2001). Post hoc power analysis: An idea whose time has passed? Pharmacotherapy, 21, 405-409.

Little, R. J. A., \& Rubin, D. B. (1987). Statistical analysis with missing data. New York: Wiley.

Little, T. D., Cunningham, W. A., Shahar, G., \& Widaman, K. F. (2002). To parcel or not to parcel: Exploring the question, weighing the merits. Structural Equation Modeling, 9, 151-173.

Lovibond, S. H., \& Lovibond, P. F. (2004). Manual for the depression anxiety stress scales (2 Ed.). Sydney: Psychology Foundation.

Lund, H. G., Reider, B. D., Whiting, A. B., \& Prichard, J. R. (2010). Sleep patterns and predictors of disturbed sleep in a large population of college students. Journal of Adolescent Health, 46, 124 132. https://doi.org/10.1016/j.jadohealth.2009.06.016.

Quested, E., Bosch, J., Burns, V. E., Cummings, J., Ntoumanis, N., \& Duda, J. L. (2011). Basic psychological need satisfaction, cognitive appraisals, and dancers' hormonal and emotional responses. Journal of Sport \& Exercise Psychology, 33, 828 - 46.

Reis, H. T., Sheldon, K. M., Gable, S. L., Roscoe, J., \& Ryan, R. M. (2000). Daily well-being: The role of autonomy, competence, and relatedness. Personality and Social Psychology Bulletin, 26, 419-435. https://doi.org/10.1177/0146167200266002.

Ryan, R. M., Bernstein, J. H., \& Brown, K. W. (2010). Weekends, work, and well-being: Psychological need satisfactions and day of the week effects on mood, vitality, and physical symptoms. Journal of Social and Clinical Psychology, 29, 95-122.

Ryan, R. M., \& Frederick, C. M. (1997). On energy, personality, and health: Subjective vitality as a dynamic reflection of well-being. Journal of Personality, 65, 529-565. https://doi. org/10.1111/j.1467-6494.1997.tb00326.x.

Sörbom, D. (1975). Detection of correlated errors in longitudinal data. British Journal of Mathematical and Statistical Psychology, 28, 138-151.

Taylor, D. J., Vatthauer, K. E., Bramoweth, A. d., \& Ruggero, C. (2013). The role of sleep in predicting college academic performance: Is it a unique predictor? Behavioral Sleep Medicine, 11, 159-172. https://doi.org/10.1080/15402002.2011.602776.

Urner, M., Tornic, J., \& Bloch, K. E. (2009). Sleep patterns in high school and university students: A longitudinal study. Chronobiology International, 26(6), 1222-1234. https://doi. org/10.1080/07420520903244600.

Watson, D., O’Hara, M. W., Simms, L. J., Kotov, R., \& Chmielewski, M. (2007). Development and validation of the Inventory of Depression and Anxiety Symptoms (IDAS). Psychological Assessment, 19, 253-268. https://doi.org/10.1637/1040-3590.19.3.253.

Weinstein, N., Khabbaz, F., \& Legate, N. (2016). Enhancing need satisfaction to reduce psychological distress in Syrian refugees. Journal of Consulting and Clinical Psychology PMID 27018533. https://doi.org/10.1037/ccp0000095.

Weinstein, N., \& Ryan, R. M. (2011). A self-determination theory approach to understanding stress incursion and responses. Stress and Health, 27, 4-17. https://doi.org/10.1002/smi.1368.

Zimmerman, L. K. (2011). Chronotype and the transition to college life. Chronobiology International, 28(10), 904-910. https://doi. org/10.3109/07420528.2011.618959.

Zunhammer, M., Eichhammer, P., \& Busch, V. (2014). Sleep quality during exam stress: The role of alcohol, caffeine and nicotine. PloS One, 9(10), e109490. https://doi.org/10.1371/journ al.pone.0109490. 